International Conference Mathematical and Computational Biology 2011

International Journal of Modern Physics: Conference Series

Vol. 9 (2012) 480-487

C World Scientific Publishing Company

DOI: $10.1142 / \mathrm{S} 2010194512005569$

\title{
SOLVING MEDICAL AKZO NOBEL PROBLEM USING FUNCTIONAL LOAD BALANCING ALGORITHM OF 4(3) DIRK METHOD
}

\author{
UMMUL KHAIR SALMA DIN \\ School of Mathematical Sciences, Faculty of Science and Technology, \\ Universiti Kebangsaan Malaysia, 43600 UKM Bangi, Selangor, Malaysia \\ ummul@ukm.my \\ FUDZIAH ISMAIL \\ Department of Mathematics, Faculty of Sciences, \\ Universiti Putra Malaysia, 43400 UPM Serdang, Selangor, Malaysia \\ fudziah_i@yahoo.com.my \\ ZANARIAH ABDUL MAJID \\ Department of Mathematics, Faculty of Sciences, \\ Universiti Putra Malaysia, 43400 UPM Serdang, Selangor, Malaysia \\ zanamajid@hotmail.com \\ ROKIAH ROZITA AHMAD \\ School of Mathematical Sciences, Faculty of Science and Technology, \\ Universiti Kebangsaan Malaysia, 43600 UKM Bangi, Selangor, Malaysia \\ rozy@ukm.my
}

\begin{abstract}
Medical Akzo Nobel problem (MEDAKZO) is known for its tenancy of incurring high computational cost. Originates from the penetration of radio-labeled antibodies into a tissue that has been infected by a tumor, the problem has been derived from a one dimensional partial differential equations to a two dimensional ordinary differential equations thus generates a large scale of problem to be solved. This paper presents the performance of a new 4(3) diagonally implicit Runge-Kutta (DIRK) method which is suitable to excellently solve MEDAKZO problem that is stiff in nature. The sparsity pattern designed on the method enable the functions evaluations to be computed simultaneously on two processors. The functional load balancing can be profitable especially in solving large problems.
\end{abstract}

Keywords: Functional load balancing; parallel; diagonally implicit Runge-Kutta method.

\section{Introduction}

Numerical analysis is the area of mathematics and computer science that has a great importance in solving many physical problems represented by mathematical models. These problems occur throughout the natural sciences, social sciences, medicine, 
engineering, and business which then are classified as linear or nonlinear and stiff or nonstiff problems. When simulating the behaviour of those systems, mathematical models often include one or more ordinary differential equations (ODEs). The solution of ODEs can be extremely expensive due to the following factors ${ }^{1}$ :

(i) the dimension of the ODEs is very large,

(ii) the evaluation of the right-hand side factor is expensive,

(iii) the interval of the integration is very long,

(iv) the ODEs must be solved repeatedly.

The primary objective in applying parallelism in numerical computation is the significant reduction in time which appears to be one of the contributing costs in computer execution. The Medical Akzo Nobel Problem is a large sparse ODE system which made it suitable to be solved by parallel implementation ${ }^{2,3}$.

\section{The Medical Akzo Nobel Problem (Medakzo)}

The Akzo Nobel research laboratories have formulated the Medical Akzo Nobel (Medakzo) Problem in their study of the penetration of radio-labeled antibodies into a tissue that has been infected by a tumor ${ }^{4}$. It is a large system of partial differential equations (PDEs) reformulated in ODEs. The study of this problem was carried out for diagnostic as well as therapeutic purposes. The problem consists of two partial differential equations. Semi-discretization of this system yields a stiff ODE. Implicit numerical methods have always been a choice when solving stiff ordinary differential equations (ODEs). However most of them are expensive to use which caused the search for efficiently implementable methods at a cheaper cost. Diagonally implicit Runge-Kutta (DIRK) methods have been considered as methods that have almost the same advantage as the implicit Runge-Kutta methods, importantly in its stability criterion. The Medakzo Problem is of the form

$$
\frac{d y}{d t}=f(t, y), \quad y(0)=g,
$$

with

$$
y \in \mathbb{R}^{2 N}, \quad 0 \leq t \leq 20 .
$$

Here, the integer $N$ is a user-supplied parameter. The function $f$ is given by

$$
\begin{aligned}
f_{2 j-1} & =\alpha_{j} \frac{y_{2 j+1}-y_{2 j-3}}{2 \Delta \zeta}+\beta_{j} \frac{y_{2 j-3}-2 y_{2 j-1}+y_{2 j+1}}{(\Delta \zeta)^{2}}-k y_{2 j-1} y_{2 j}, \\
f_{2 j} & =-k y_{2 j} y_{2 j-1},
\end{aligned}
$$


where

$$
\begin{aligned}
& \alpha_{j}=\frac{2(j \Delta \zeta-1)^{3}}{c^{2}}, \\
& \beta_{j}=\frac{(j \Delta \zeta-1)^{4}}{c^{2}} .
\end{aligned}
$$

Here, $j$ ranges from 1 to $N, \Delta \zeta=\frac{1}{N}, y_{-1}(t)=\phi(t), y_{2 N+1}=y_{2 N-1}$ and $g \in \mathbb{R}^{2 N}$ is given by

$$
g=\left(0, v_{0}, 0, v_{0}, \ldots, 0, v_{0}\right)^{T} .
$$

The function $\phi$ is given by

$$
\phi(t)= \begin{cases}2 & \text { for } t \in(0,5], \\ 0 & \text { for } t \in(5,20]\end{cases}
$$

which means that $f$ undergoes a discontinuity in time at $t=5$. Suitable values for the parameter $k, v_{0}$ and $c$ are 100, 1 and 4, respectively. Refs. 2-5 used this problem to test the efficiency of their algorithms.

\section{The 4(3) DIRK Method}

In this paper, we present an efficient fourth order DIRK method with A-stability which is suitable for solving stiff ODEs. This method has an embedded method of lower order which is used to estimate the local error and to select the step size according to a prescribed tolerance without additional costs. The method is designed according to the sparsity structure as discussed in Refs. 6 and 7 which permits the method to be implemented on two processors simultaneously. The basic idea of parallelism in this method is to have every two consecutive functions evaluations independent of each other therefore each of them could be evaluated on a different processor. The parallelization of this method is based on functional parallelism which is applied when there are independent tasks working on different operations to different data elements. A digraph as shown in Fig.1 is used to model the sparsity pattern for a Runge-Kutta method having four function evaluations where parallel implementation on two processors is possible. $K_{l}, K_{2}, K_{3}$ and $K_{4}$ represent the function evaluations in a Runge Kutta method. 


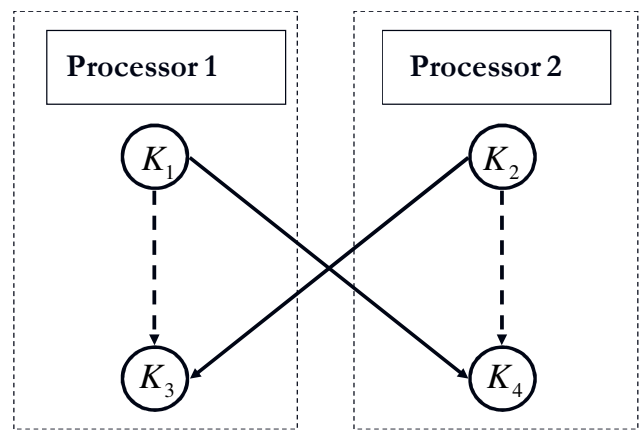

Fig. 1. Digraph of Runge Kutta method for two processors implementation.

An embedded Runge-Kutta method is a method in which two Runge-Kutta estimates are obtained using the same auxiliary functions $K_{i}$ but with a different linear combination of these functions so that one estimate has an order one greater than the other. This will imply the required computation effort is minimized since there is no extra function evaluation.

Consider two Runge-Kutta formulae with orders $p$ and $q$ where $q=p-1$ which have common function evaluations. Assume $y^{p}{ }_{n+1}$ and $y_{n+1}^{q}$ are estimates of $y\left(x_{n+1}\right)$, where $x_{n+1}=x_{n}+h_{n}$. If the $p$ th order method

$$
y_{n+1}^{p}=y_{n}+h_{n} \sum_{i=1}^{s} b_{i} K_{i}
$$

we would seek to find a $\hat{b}$ such that

$$
y_{n+1}^{q}=y_{n}+h_{n} \sum_{i=1}^{r} \hat{b}_{i} K_{i}
$$

where

$$
K_{i}=f\left(x_{n}+c_{i} h, y_{n}+h_{n} \sum_{j=1}^{i} a_{i j} K_{j}\right) \quad i=1,2, \ldots, s,
$$

with $s$ and $r$ being the number of stages.

The principal local truncation error in the basic DIRK method could be estimated by:

$$
T_{n+1}=y^{p}{ }_{n+1}-y^{q}{ }_{n+1}
$$

In this work, Runge-Kutta embedding in local extrapolation (or higher order) mode is considered where the lower order formula being used solely for local error estimation and 
hence stepsize control. The higher order estimates of $y\left(x_{n+1}\right)$ will advances the integration and determines the accuracy.

The derivation of Runge Kutta 4(3) pair with four stages will not yield an A-stable method $^{6}$. Therefore in order to seek for A-stability we added two more stages to the sparsity pattern which gave more number of the unknowns. As for the error estimator a third order Runge Kutta method with four stages is considered. For convenience the method is labeled as P2DIRK4(3).

For the embedding method to be of fourth order, eight conditions need to be fulfilled $^{8}$ :

$$
\begin{aligned}
& \sum b_{i}=1 \\
& \sum b_{i} c_{i}=\frac{1}{2} \\
& \sum b_{i} c_{i}^{2}=\frac{1}{3} \\
& \sum b_{i} a_{i j} c_{j}=\frac{1}{6} \\
& \sum b_{i} c_{i}^{3}=\frac{1}{4} \\
& \sum b_{i} c_{i} a_{i j} c_{j}=\frac{1}{8} \\
& \sum b_{i} a_{i j} c_{j}^{2}=\frac{1}{12} \\
& \sum b_{i} a_{i j} a_{j k} c_{k}=\frac{1}{24}
\end{aligned}
$$

The coefficients for P2DIRK4(3) are given in Fig. 2. 


\begin{tabular}{l|lllllll}
$\frac{1}{2}$ & $\frac{1}{2}$ & & & & & \\
$\frac{1}{3}$ & 0 & $\frac{1}{3}$ & & & & \\
$\frac{1}{5}$ & $\frac{3}{25}$ & $-\frac{21}{50}$ & $\frac{1}{2}$ & & & \\
$\frac{7}{9}$ & $-\frac{17}{27}$ & $\frac{29}{27}$ & 0 & $\frac{1}{3}$ & & \\
$\frac{5}{6}$ & $-\frac{7597}{4680}$ & $\frac{82471}{37440}$ & $-\frac{323}{936}$ & $\frac{19}{192}$ & $\frac{1}{2}$ & \\
1 & $-\frac{19}{15}$ & $\frac{14}{15}$ & $\frac{1}{2}$ & $\frac{1}{2}$ & 0 & $\frac{1}{3}$ \\
\hline 0 & 0 & $\frac{1875}{3952}$ & $\frac{81}{104}$ & $-\frac{6}{19}$ & $\frac{1}{16}$ \\
0 & 0 & $\frac{25}{52}$ & $\frac{27}{52}$ & &
\end{tabular}

Fig. 2. The P2DIRK4(3).

The method is found to be A-stable with A-stable error estimators.

\section{Numercal Experiments}

The Medakzo problem is tested using P2DIRK4(3) by comparing the sequential and parallel time taken to solve the problems with various dimensions $(N)$ using tolerance $10^{-2}, 10^{-4}$ and $10^{-6}$. Table 1 shows the execution time (in second) for both sequential and parallel execution together with the speedup and efficiency measures.

Table 1. The speedup and efficiency P2DIRK4(3) in solving the Medakzo Problem

\begin{tabular}{cccccc}
\hline TOL & $\mathrm{N}$ & $\begin{array}{c}\text { Sequential } \\
\text { Time }\end{array}$ & $\begin{array}{c}\text { Parallel } \\
\text { Time }\end{array}$ & Speedup & Efficiency \\
\hline \multirow{6}{*}{$10^{-2}$} & 20 & 0.179 & 0.133 & 1.35 & 67.25 \\
& 60 & 3.573 & 2.375 & 1.50 & 75.21 \\
& 100 & 14.726 & 9.909 & 1.49 & 74.31 \\
& 150 & 54.945 & 36.769 & 1.49 & 74.72 \\
& 200 & 135.967 & 89.516 & 1.52 & 75.95 \\
& 300 & 453.027 & 288.651 & 1.57 & 78.47 \\
\hline
\end{tabular}


Table 1. (Continued)

\begin{tabular}{cccccc}
\hline TOL & N & $\begin{array}{c}\text { Sequential } \\
\text { Time }\end{array}$ & $\begin{array}{c}\text { Parallel } \\
\text { Time }\end{array}$ & Speedup & Efficiency \\
\hline \multirow{4}{*}{$10^{-4}$} & 20 & 0.510 & 0.376 & 1.36 & 67.82 \\
& 60 & 9.756 & 6.309 & 1.55 & 77.32 \\
& 100 & 43.363 & 27.381 & 1.58 & 79.19 \\
& 150 & 148.808 & 91.638 & 1.62 & 81.19 \\
& 200 & 362.187 & 220.535 & 1.64 & 82.12 \\
& 300 & 1178.651 & 702.638 & 1.68 & 83.87 \\
& & & & & \\
& 20 & 1.282 & 0.909 & 1.41 & 70.52 \\
& 60 & 27.726 & 16.948 & 1.64 & 81.80 \\
& 100 & 132.227 & 79.414 & 1.67 & 83.25 \\
& 150 & 473.907 & 279.260 & 1.70 & 84.85 \\
& 200 & 1152.249 & 668.582 & 1.72 & 86.17 \\
& 300 & 3742.193 & 2111.490 & 1.77 & 88.61 \\
& & & & \\
\hline
\end{tabular}

Graph showing the relation between the dimensions of the problems and the speedups for every tolerance used is displayed in Fig. 3.

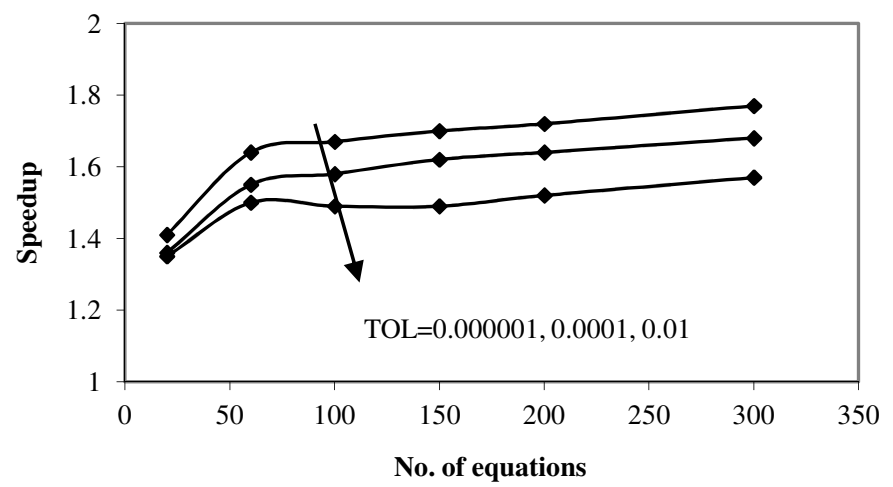

Fig. 3. The Speedup of P2DIRK4(3) when Solving Medakzo Problem. 


\section{Discussion and Conclusion}

From Table 1, the maximum speedup in the experiment for Medakzo Problem is 1.77 with efficiency almost $89 \%$. The performances for dimensions beyond 300 for the problem have not been observed because the experiments are time consuming. Medakzo Problem is a nonlinear stiff problem. In the execution process for the problem it has to pass through a number of routes in the parallel code in order to complete the integration. These have influenced the timing because several processes are repeated. It could also be observed that for higher tolerance, the speedup in solving the same number of equations is better. In general, the time reduction by using the parallel implementation with P2DIRK4(3) is very significant when solving the problem compared to the sequential implementation.

\section{Acknowledgments}

The first author thankfully acknowledged UKM for providing the Geran Galakan Penyelidik Muda (UKM-GGPM-ICT-121-2010) and Ministry of Higher Education, Malaysia for the financial support received for this study.

\section{References}

1. M.A. Kartawidjaja, H. Suhartanto and T. Basaruddin, Performance of a parallel technique for solving stiff ODEs using a block-diagonal implicit Runge-Kutta method, in The IPSI BgD Transactions on Advanced Research, 1, 2 (2001), p. 68-73.

2. J. M. Bahi, S. Contassot-Vivier and R. Couturier, Asynchronism for iterative algorithms in global computing environment,in Proc of the $16^{\text {th }}$ Annual Int. Sympsium on High Performance Computing Systems and Applications (HPC'02), (2002).

3. M. Korch and T. Rauber, Simulation-based Analysis of parallel Runge-Kutta solvers, in PARA 2004, LNCS 3732, eds. J. Dongarra, K. Madsen and J. Waśniewski (Springer-Verlag, Berlin, 2006), p. 1105-1114.

4. F. Mazzia, C. Magherini and F. Iavernaro, Test set for initial value problems solvers, in Report 43/2006 (Department of Mathematics, University of Bari, Italy, 2006).

5. M. Korch and T. Rauber, Applicability of Load Balancing Strategies to Data-Parallel Embedded Runge-Kutta Integrators, in Euro-Par 2006, LNCS 4128, eds. W.E. Nagel et al. (Springer-Verlag, Berlin, 2006,) p. 720-729.

6. A. Iserles, and S.P. Nørsett, On the theory of parallel Runge-Kutta methods, in IMA Journal of Numerical Analysis, 10 (1990), p. 463-488.

7. K.R. Jackson, and S.P. Nørsett, The potential for parallelism in Runge-Kutta methods. Part1: RK formulas in standard form, in Siam J. Numer. Anal., 32, 1 (1995), p. 49-82.

8. J.C. Butcher, Numerical methods for ordinary differential equations (John Wiley \& Sons, 2003). 